\title{
COVERING IN THE LATTICE OF SUBUNIVERSES OF A FINITE DISTRIBUTIVE LATTICE
}

\author{
ZSOLT LENGVÁRSZKY and GEORGE F. MCNULTY
}

(Received 3 February 1997; revised 30 August 1998)

Communicated by B. A. Davey

\begin{abstract}
The covering relation in the lattice of subuniverses of a finite distributive lattice is characterized in terms of how new elements in a covering sublattice fit with the sublattice covered. In general, although the lattice of subuniverses of a finite distributive lattice will not be modular, nevertheless we are able to show that certain instances of Dedekind's Transposition Principle still hold. Weakly independent maps play a key role in our arguments.
\end{abstract}

1991 Mathematics subject classification (Amer. Math. Soc.): primary 06B05.

Keyinords and phrases: distributive lattice, sublattice, covering, maximal sublattice.

\section{Introduction}

For any lattice $\mathbf{L}$ the set $\operatorname{Sub} \mathbf{L}$ of subuniverses of $\mathbf{L}$ is lattice-ordered by set-inclusion. In general, properties of $\mathbf{L}$ are not likely to hold for $\operatorname{Sub} \mathbf{L}$ and the structure of $\operatorname{Sub} \mathbf{L}$ may not be clear even if the structure of $\mathbf{L}$ itself is transparent. For example, if $\mathbf{L}$ is not a chain, then $\operatorname{Sub} \mathbf{L}$ is not even modular, as can be easily seen since $\mathbf{N}_{5}$ is embeddable in Sub $2 \times 2$. Even for the well-understood class of finite distributive lattices not much seems to be known about the corresponding lattices of subuniverses.

Let $\mathbf{K}$ be a sublattice of $\mathbf{L}$. We say $x \in L$ splits a covering of $\mathbf{K}$ provided there are $a, b \in K$ so that $b$ covers $a$ in $\mathbf{K}$ but $a<x<b$ in $\mathbf{L}$. We say $x \in L$ completes a square over $\mathbf{K}$ provided there are $a, b, c \in K$ with $a<c<b$ so that $x \vee c=b$ and $x \wedge c=a$. Further, we say $x$ completes a covering square over $\mathbf{K}$ if, in addition, $a \prec c \prec b$ in $\mathbf{K}$. The length of the lattice $\mathbf{K}$ is the least upper bound on the length of chains in $\mathbf{K}$. The length of the trivial lattice with only one element is 0 , while the

The first author was supported in part by Hungarian NSF Research Grant No. F7525.

(C) 1998 Australian Mathematical Society $0263-6115 / 98 \$ A 2.00+0.00$ 
length of the two-element lattice is $1 . \ell(\mathbf{K})$ denotes the length of $\mathbf{K} . \mathrm{Sg}^{\mathbf{L}} X$ denotes the subuniverse of $\mathbf{L}$ generated by the set $X$. The theorem below may be folklore.

THEOREM 1. Let $\mathbf{L}$ be a lattice of finite height. Suppose that $\mathbf{K}$ and $\mathbf{K}^{*}$ are sublattices of $\mathbf{L}$ such that $\mathbf{K}^{*}$ covers $\mathbf{K}$ in Sub $\mathbf{L}$. Then one of the following cases happens: (1) $K^{*}=K \cup\left\{1^{\mathbf{K}^{*}}\right\}$.

(2) $K^{*}=K \cup\left\{0^{K^{*}}\right\}$.

(3) There is an element of $K^{*}-K$ which splits a covering of $\mathbf{K}$.

(4) There is an element of $K^{*}-K$ which completes a square over $\mathbf{K}$.

Moreover, if $\mathbf{L}$ is modular, then in Case 4 we can insist that the element completes a covering square over $\mathbf{K}$.

Proof. Suppose that neither Case 1 nor Case 2 holds. Then for every $x \in K^{*}-K$ there must be $a, b \in K$ so that $a<x<b$. Among all possible choices of $x \in K^{*}-K$ and $a, b \in K$ with $a<x<b$, make a choice so that the length of the interval from $a$ to $b$ measured in $\mathbf{K}$ is as small as possible. If this interval has length 1 in $\mathbf{K}$, then $b$ covers $a$ in $\mathbf{K}$ and $x$ splits a covering in $\mathbf{K}$; that is, Case 3 holds. So consider the case when the chosen interval has length greater than 1 . Pick any $c \in K$ with $a<c<b$. From the minimality of our choice, it follows that $a=c \wedge x$ and $c \vee x=b$. We conclude that $x$ completes a square over $\mathbf{K}$. This gives Case 4 . Moreover, since $\mathbf{N}_{5}$ cannot be a sublattice of $\mathbf{L}$ if $\mathbf{L}$ is modular, we conclude that $b$ covers $c$ covers $a$ in $\mathbf{K}$, in that case.

In general, the configurations described in Theorem 1 do not always lead to coverings between the two sublattices. Moreover, the situations described in Cases 3 and 4 of that theorem can occur for the same covering pair $\mathbf{K} \subseteq \mathbf{K}^{*}$. For each lattice in Figure 1 , let $\mathbf{K}$ be the sublattice with points indicated by $\bullet$. In $\mathbf{L}_{0}, x$ splits a covering in $\mathbf{K}$, but $\mathbf{K} \vee\{x\}$ does not cover $\mathbf{K}$. In $\mathbf{L}_{1}, x$ completes a square over $\mathbf{K}$, but $\mathbf{K} \vee\{x\}$ does not cover $\mathbf{K}$. $\mathbf{K}$ is a maximal sublattice of $\mathbf{L}_{2}$, but $x$ splits a covering in $\mathbf{K}$ while $y$ completes a square over $\mathbf{K}$.
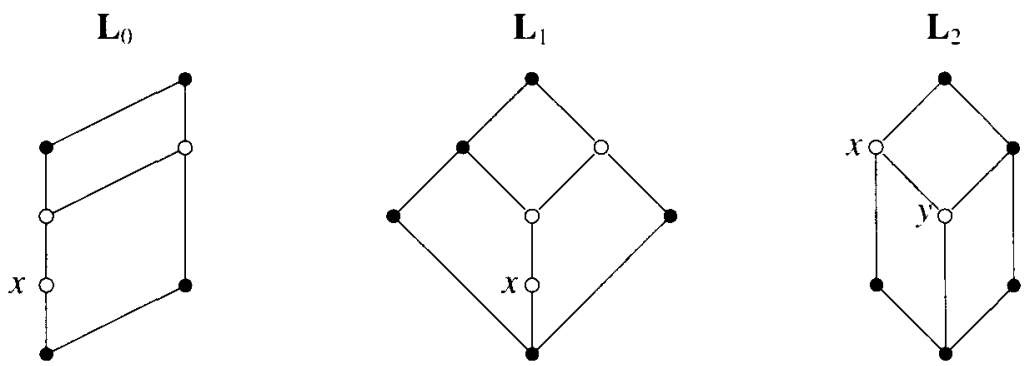

FIGURE 1. Three lattices witnessing the ambiguity of Theorem 1 
In the case of finite distributive lattices, we prove a converse of Theorem 1:

THEOREM 2. Let $\mathbf{L}$ be a finite distributive lattice, let $\mathbf{K} \in \operatorname{Sub} \mathbf{L}$, and let $x \in L-K$ with $\mathbf{K}^{*}=\operatorname{Sg}^{\mathrm{L}}(K \cup\{x\})$.

(1) If $x=0^{\mathbf{K}^{*}}$, then $\mathbf{K}^{*}$ covers $\mathbf{K}$ and $\ell\left(\mathbf{K}^{*}\right)=1+\ell(\mathbf{K})$.

(2) If $x=1^{\mathbf{K}^{*}}$, then $\mathbf{K}^{*}$ covers $\mathbf{K}$ and $\ell\left(\mathbf{K}^{*}\right)=1+\ell(\mathbf{K})$.

(3) If $x$ splits a covering in $\mathbf{K}$, then $\mathbf{K}^{*}$ covers $\mathbf{K}$ and $\ell\left(\mathbf{K}^{*}\right)=1+\ell(\mathbf{K})$.

(4) If $x$ completes a square over $\mathbf{K}$, then $\ell\left(\mathbf{K}^{*}\right)=\ell(\mathbf{K})$. If $x$ completes a covering square over $\mathbf{K}$, then $\mathbf{K}^{*}$ covers $\mathbf{K}$ as well.

The proof of this theorem occupies Section 3 below. Our primary tool in proving this theorem is a representation theorem (Theorem 3) for lattices of the form Sub $\mathbf{L}$, where $\mathbf{L}$ is a finite distributive lattice. We also show in Theorem 4 that certain instances of Dedekind's Transposition Principle hold in Sub $\mathbf{L}$, where $\mathbf{L}$ is a finite distributive lattice. Theorem 4 can be viewed as an extension of Theorem 2. Theorems 1, 2, and 3 are drawn from the first author's Ph.D. dissertation [13]. The direct proof of Theorem 3 given there differs from the one presented below.

The investigation of covering relations in $\operatorname{Sub} \mathbf{L}$ is, in essence, an investigation of maximal sublattices. One of the earliest contributions to the study of maximal sublattices of distributive lattices was made in 1952 by Hashimoto [11]. During the 1970's the work of Adams [2], Chen, Koh and Tan [8], and Rival [18, 19], carried these investigations forward. In recent years, Abad and Adams [1], Adams, Dwinger and Schmid [3], Ryter and Schmid [20], and Vogt [22] have added considerably to our understanding of maximal sublattices of finite distributive lattices. In addition, the recent paper of Adams, Freese, Nation and Schmid [4] examines maximal sublattices of finite bounded lattices, a class which includes the finite distributive lattices. In a different direction, Tüma [21] has characterized the covering relation in the ordered set of distributive subsemilattices of a finite distributive semilattice. The connection here is that finite distributive semilattices are those algebras that result from eliminating the meet operation from a finite distributive lattice.

Underlying all these approaches is a characterization of the maximal sublattices of a (finite) distributive lattice. In Section 5, we compare the characterization present in Theorems 1 and 2 and the characterization arising in the sequence of papers cited above.

The authors would like to thank the referee for suggesting the use of the lattice of closure operators in the proof of Theorem 3 and for pointing out the connection between weakly independent maps and topological closure operators in distributive lattices. The proof of Theorem 3 offered in the first version of this paper, which also differed from that given by the first author in [13], was more involved conceptually, and relied on a Galois connection. We are also grateful that the referee brought Tưma's 
paper to our attention.

\section{The representation}

Let $\mathbf{L}$ be a finite lattice. Let $0^{\mathbf{L}}$ and $1^{\mathbf{L}}$ denote the least and the greatest elements of $\mathbf{L}$ and let $J_{0}(\mathbf{L})$ denote the set of join irreducible elements of $\mathbf{L}$, including $0^{\mathbf{L}}$. We define

$$
\mathscr{S}_{\mathbf{L}}=\left\{K: K \text { is a sublattice of } \mathbf{L} \text { and } 1^{\mathrm{L}} \in K\right\}
$$

Evidently, $\mathscr{S}_{\mathbf{L}}$ is a sublattice of the lattice Sub $\mathbf{L}$ of all subuniverses of $\mathbf{L}$. In fact, an understanding of the covering relation in $\mathscr{S}_{\mathrm{L}}$ leads directly to an understanding of the covering relation in $\operatorname{Sub} \mathbf{L}$.

A map $\varphi: J_{0}(\mathbf{L}) \rightarrow L$ is said to be weakly independent provided

$$
\varphi(j)=\bigwedge\left\{\varphi(k): k \in J_{0}(\mathbf{L}) \text { and } j \leq \varphi(k)\right\}, \text { for all } j \in J_{0}(\mathbf{L}) .
$$

In other words, $\varphi(j)$ is the smallest member of the range of $\varphi$ at least as large as $j$. Hence,

- $j \leq \varphi(j)$ for each $j \in J_{0}(\mathbf{L})$ and each weakly independent map $\varphi$;

- any two weakly independent maps with the same ranges are the same.

We denote the set of all weakly independent maps of $\mathbf{L}$ by $\mathscr{W}_{\mathbf{L}}$. This set can be made into a lattice by setting $\varphi \leq \psi$ if and only if $\varphi(j) \leq \psi(j)$ for all $j \in J_{0}(\mathbf{L})$. Then meets work out so that $(\varphi \wedge \psi)(j)=\varphi(j) \wedge \psi(j)$. The map $\varphi_{1}$ on $J_{0}(\mathbf{L})$ which is constantly $1^{\mathrm{L}}$ is a weakly independent map, and it is the largest element of $\mathscr{W}_{\mathbf{L}}$. Hence, joins also exist in $\mathscr{W}_{\mathbf{L}}$.

We show in this section that $\mathscr{S}_{\mathbf{L}}$ and $\mathscr{W}_{\mathbf{L}}$ are dually isomorphic, provided $\mathbf{L}$ is a finite distributive lattice. This dual isomorphism involves another lattice: the lattice $\mathscr{K}_{\mathbf{L}}$ of all topological closure operators on $\mathbf{L}$. Our representation involves two steps. First, it is part of the early lore of lattice theory that $\mathscr{S}_{\mathbf{L}}$ is dually isomorphic to $\mathscr{K}_{\mathbf{L}}$. Second, as we will show here, restriction to $J_{0}(\mathbf{L})$ is an isomorphism from $\mathscr{K}_{\mathrm{L}}$ onto $\mathscr{W}_{\mathbf{L}}$.

A unary operation $\gamma: L \rightarrow L$ is called a closure operator on $\mathbf{L}$ provided

(C1) $x \leq \gamma(x)$, for all $x \in L$,

(C2) $\gamma(\gamma(x))=\gamma(x)$, for all $x \in L$, and

(C3) if $x \leq y$, then $\gamma(x) \leq \gamma(y)$, for all $x, y \in L$.

A closure operator $\gamma$ is said to be topological if it further satisfies

(C4) $\gamma(x \vee y)=\gamma(x) \vee \gamma(y)$, for all $x, y \in L$.

Observe that condition (C4) is stronger than (C3). 
An early treatment of closure operators (for lattices of sets ordered by inclusion) can be found in Moore's 1910 treatise [15] on analysis. Condition (C4) is drawn from Kuratowski's 1933 exposition [12] of general topology (it should be noted that Kuratowski also insisted that $\gamma(0)=0$, a condition that is not adopted here). It was Moore who established (in his context) the natural one-to-one correlation between the collection of subsets of $L$ which include 1 and are closed under arbitrary meets and the collection of all closure operators. Moore's reasoning applies with no essential changes to arbitrary complete lattices, see for example Birkhoff [7]. More recent expositions can be found in Balbes and Dwinger [5] and in Davey and Priestley [10]. Ward [23] in 1942 proved that the closure operators on a complete lattice constituted a lattice with respect to the coordinate-wise ordering. Other early related works by Ore are $[16,17]$.

Here are the salient points from the lore of closure operators. For the time being, let $\mathbf{L}$ be any complete lattice. By $\mathscr{M}_{\mathbf{L}}$ we denote the collection of all subsets $X$ of $L$ such that $1^{\mathrm{L}} \in X$ and $X$ is closed under arbitrary (even infinite) meets. By $\mathscr{C}_{\mathbf{L}}$ we denote the collection of all closure operators on $\mathbf{L}$. By $\mathscr{K}_{\mathbf{L}}$ we denote the collection of all topological closure operators on $\mathbf{L}$. Let $\Psi: \mathscr{M}_{\mathbf{L}} \rightarrow \mathscr{C}_{\mathbf{L}}$ be the function defined so that

$$
\Psi_{X}(a)=\bigwedge\{x \in X: a \leq x\} \text { for all } X \in \mathscr{M}_{\mathbf{L}} \text { and all } a \in L .
$$

Let $C: \mathscr{C}_{\mathbf{L}} \rightarrow \mathscr{M}_{\mathbf{L}}$ be the function defined by

$$
C_{\gamma}=\{a \in L: \gamma(a)=a\} .
$$

It is part of the lore (and also easily proven) that $\Psi$ and $C$ are well-defined and that they are inverses of each other. Now $\mathscr{M}_{\mathrm{L}}$ is lattice-ordered by set inclusion and the order that $\mathscr{C}_{\mathbf{L}}$ inherits from $\mathrm{L}^{L}$ is a lattice ordering of $\mathscr{C}_{\mathbf{L}}$. The meets in these lattices are the expected ones, and the joins, as usual, are defined via meets. The maps $\Psi$ and $C$ are dual isomorphisms between these lattices. Let $\Psi^{\prime}$ denote the restriction of $\Psi$ to $\mathscr{I}_{\mathbf{L}}$ and let $C^{\prime}$ denote the restriction of $C$ to $\mathscr{K}_{\mathbf{L}}$. It is not hard to see that $\mathscr{S}_{\mathbf{L}}$ and $\mathscr{K}_{\mathbf{L}}$ are lattices and that the maps $\Psi^{\prime}$ and $C^{\prime}$ are inverse dual isomorphisms between these two lattices.

THE EXTENSION LeMma. Let $\mathbf{L}$ be a finite distributive lattice. Every weakly independent map of $\mathbf{L}$ extends to a unique topological closure operator on $\mathbf{L}$. The restriction of any closure operator (topological or not) of $\mathbf{L}$ to $J_{0}(\mathbf{L})$ is a weakly independent map of $\mathbf{L}$.

PROOF. First suppose that $\varphi$ is weakly independent. We define $\gamma: L \rightarrow L$ via

$$
\gamma(x)=\bigvee_{j \in N} \varphi(j)
$$


where $N$ is the set of all join irreducibles $j$ such that $j \leq x$. This is the only possible way to extend $\varphi$ to a join-preserving map from $L$ into $L$.

Now $x \leq \gamma(x)$, since $j \leq \varphi(j)$ for each $j \in J_{0}(\mathbf{L})$.

To see that $\gamma$ preserves joins, let $x, y \in L$ with $x=\bigvee N$ and $y=\bigvee M$, where $N$ and $M$ are, respectively, the sets of join irreducibles below $x$ and $y$. Now suppose $j$ is join irreducible and $j \leq x \vee y$. Since $\mathbf{L}$ is distributive $j$ is join prime. So $j \leq x$ or $j \leq y$. This means that $M \cup N$ is the set of all join irreducibles $j$ such that $j \leq x \vee y$. So

$$
\gamma(x \vee y)=\bigvee_{j \in \mathcal{N}} \varphi(j) \vee \bigvee_{j \in M} \varphi(j)=\gamma(x) \vee \gamma(y)
$$

To complete the argument that $\gamma$ is a topological closure operator, we need to verify that it is idempotent. Notice that

$$
\gamma(\gamma(x))=\bigvee_{j \in N}(\gamma(\varphi(j)))
$$

where $x=\bigvee N$ and $N$ is the set of join irreducibles below $x$. Let $M_{j}$ be the set of join irreducibles below $\varphi(j)$. Since $\varphi$ is weakly independent, we have $\varphi(k) \leq \varphi(j)$ for each $k \in M_{j}$. This entails that $\gamma(\varphi(j))=\varphi(j)$ since

$$
\varphi(j) \leq \gamma(\varphi(j))=\bigvee_{k \in M_{i}} \varphi(k) \leq \varphi(j) .
$$

Consequently, $\gamma(\gamma(x))=\gamma(x)$ as desired. We conclude that $\gamma$ is a topological closure operator.

Now suppose that $\gamma$ is any closure operator on $\mathbf{L}$ and let $\varphi$ denote the restriction of $\gamma$ to $J_{0}(\mathbf{L})$. Let $j \in J_{0}(\mathbf{L})$ and let $M=\left\{\varphi(k): k \in J_{10}(\mathbf{L})\right.$ and $\left.j \leq \varphi(k)\right\}$. Since $j \leq \gamma(j)=\varphi(j)$, we see that $\varphi(j) \in M$. Suppose that $\varphi(k)$ is an arbitrary element of $M$ (so that $k \in J_{0}(\mathbf{L})$ and $\left.j \leq \varphi(k)\right)$. But then

$$
\varphi(j)=\gamma(j) \leq \gamma(\varphi(k))=\gamma(\gamma(k))=\gamma(k)=\varphi(k) .
$$

Therefore $\varphi(j)$ is the least element of $M$. This means that $\varphi$ is a weakly independent map.

With every subset $X \subseteq L$, we can associate a weakly independent map $\Phi_{X}$ defined via

$$
\Phi_{X}(j)=\bigwedge\{x \in X: j \leq x\}
$$

for all $j \in J_{0}(\mathbf{L})$. Further, we can construe $\Phi$ as a map from the power set of $L$ into $\mathscr{W}_{\mathrm{L}}$ which assigns to $X \subseteq L$ the map $\Phi_{X}$. 
In the reverse direction, for each weakly independent $\operatorname{map} \varphi$ we can associate a sublattice $K_{\varphi}$ of $\mathbf{L}$, namely the sublattice of $\mathbf{L}$ generated by the range of $\varphi$. Further, we can regard $K$ as a map from $\mathscr{W}_{\mathbf{L}}$ into $\operatorname{Sub} \mathbf{L}$. Now notice that $\mathbf{1}^{\mathbf{L}}=\bigvee J_{0}(\mathbf{L})=$ $\vee \operatorname{Range}(\varphi)$. This means that $K$ is a map from $\mathscr{W}_{\mathbf{L}}$ into $\mathscr{S}_{\mathbf{L}}$.

THEOREM 3. Let $\mathbf{L}$ be a finite distributive lattice. Then $\Phi$ and $K$ are inverses of each other, establishing dual isomorphisms between $\mathscr{S}_{\mathbf{L}}$ and $\mathscr{W}_{\mathbf{L}}$. Also $J_{0}\left(K_{\varphi}\right)=\varphi\left(J_{0}(\mathbf{L})\right)$, and consequently $1+\ell\left(K_{\psi}\right)=|\operatorname{Range}(\varphi)|$ for all $\varphi \in \mathscr{W}_{\mathbf{L}}$. Moreover, $\Phi_{X}=\Phi_{Y}$ whenever $X$ and $Y$ generate the same sublattice of $\mathbf{L}$.

PROOF. We summarize the information from the Extension Lemma and the lore of closure operators in the following display:

$$
\begin{aligned}
& \mathscr{S}_{\mathbf{L}} \stackrel{\Psi}{\longrightarrow} \mathscr{K}_{\mathbf{L}} \stackrel{\rho}{\longrightarrow} \mathscr{W}_{\mathbf{L}} \\
& \mathscr{S}_{\mathbf{L}} \stackrel{C^{\prime}}{\longleftarrow} \mathscr{K}_{\mathbf{L}} \stackrel{\varepsilon}{\longleftarrow} \mathscr{W}_{\mathbf{L}}
\end{aligned}
$$

where $\rho$ denotes restriction to $J_{0}(\mathbf{L})$ and $\varepsilon$ denotes extension to $L$.

The maps $\rho \circ \Psi^{\prime}$ and $C^{\prime} \circ \varepsilon$ are inverses of each other, and establish dual isomorphism between $\mathscr{S}_{\mathbf{L}}$ and $\mathscr{W}_{\mathbf{L}}$. Evidently, $\Phi=\rho \circ \Psi^{\prime}$. To see that $K=C^{\prime} \circ \varepsilon$, suppose that $\varphi \in \mathscr{W}_{\mathbf{L}}$ and that $\gamma$ is the extension of $\varphi$ to a topological closure operator on $\mathbf{L}$. We need to demonstrate that $\{a \in L: \gamma(a)=a\}$ is the subuniverse $K_{\varphi}$ of $\mathbf{L}$ generated by the range of $\varphi$. Since $\gamma(\varphi(j))=\gamma(\gamma(j))=\gamma(j)=\varphi(j)$, for all $j \in J_{0}(\mathbf{L})$, we see that the range of $\varphi$ is included in $\{a \in L: \gamma(a)=a\}$. Since this latter set is a subuniverse, we know that $K_{\varphi} \subseteq\{a \in L: \gamma(a)=a\}$. The reverse inclusion follows from the fact that $\gamma$ is a join-preserving map extending $\varphi$ and the fact that every member of $L$ is a join of join irreducible elements. Consequently, $\Phi$ and $K$ are inverses of each other, and they establish a dual isomorphism between $\mathscr{S}_{\mathbf{L}}$ and $\mathscr{W}_{\mathbf{L}}$.

Now, suppose $\varphi \in \mathscr{W}_{\mathbf{L}}$. To see that $J_{0}\left(K_{\varphi}\right)=\varphi\left(J_{0}(\mathbf{L})\right)$, we establish the two inclusions.

First suppose $k$ is a join irreducible member of $K_{\varphi}$. Decompose $k$ as a join of elements join irreducible in $\mathbf{L}: k=\bigvee N$, where $N \subseteq J_{0}(\mathbf{L})$. Now

$$
k=\gamma(k)=\gamma\left(\bigvee_{j \in N} j\right)=\bigvee_{j \in N} \gamma(j)
$$

Since $\gamma(j) \in K_{\varphi}$ for all $j \in N$, and since $k$ is a join irreducible element of $\mathbf{K}_{\varphi}$, it must be that $k=\gamma(j)=\varphi(j)$ for some $j \in N$. Therefore, $k \in \varphi\left(J_{0}(\mathbf{L})\right)$, establishing the first inclusion.

For the reverse inclusion take $j$ to be a join irreducible element of $\mathbf{L}$. We wish to prove that $\varphi(j)$ is join irreducible in $K_{\varphi}$. So let $\varphi(j)=\bigvee M$ where $M \subseteq J_{0}\left(\mathbf{K}_{\varphi}\right)$. 
Since $j \leq \varphi(j)$, we know that $j \leq \bigvee M$. But $j$, being join irreducible, is join prime. So pick $m \in M$ such that $j \leq m$. But then $\varphi(j)=\gamma(j) \leq \gamma(m)=m$. By the choice of $M$, we have $m \leq \varphi(j)$ as well. So $\varphi(j)=m \in M \subseteq J_{0}\left(\mathbf{K}_{\varphi}\right)$. Consequently, the second inclusion is established.

Finally, suppose that $X$ and $Y$ generate the same sublattice of $\mathbf{L}$. We want to show that $\Phi_{X}=\Phi_{Y}$. To simplify notation, let $\varphi=\Phi_{X}$ and $\psi=\Phi_{Y}$. Let $\gamma$ denote the unique extension of $\varphi$ to a topological closure operator. In view of the definition of $\Phi_{X}$ we see that $\varphi(j)$ is in the sublattice generated by $X \cup\left\{1^{\mathrm{L}}\right\}$, for all $j \in J_{0}(\mathbf{L})$. Hence $K_{\psi}$ is included in that sublattice as well. For the reverse inclusion, let $x \in X$. Let $N \subseteq J_{0}(\mathbf{L})$ so that $x=\bigvee N$. Then

$$
x \leq \gamma(x)=\bigvee_{j \in N} \varphi(j) \leq x,
$$

where the last inequality follows from the definition of $\varphi$. Consequently, $x=\gamma(x)$ for all $x \in X$. This entails that $X \subseteq K_{\varphi}$. Therefore, $\mathbf{K}_{\varphi}$ is the sublattice of $\mathbf{L}$ generated by $X \cup\left\{\mathbf{l}^{\mathbf{L}}\right\}$. Likewise, $\mathbf{K}_{\psi}$ is the sublattice of $\mathbf{L}$ generated by $Y \cup\left\{\mathbf{l}^{\mathbf{L}}\right\}$. Hence, $K_{\psi}=K_{\psi}$. But $K$ is a one-to-one map. It follows that $\Phi_{X}=\varphi=\psi=\Phi_{Y}$, as desired.

A similar dual isomorphism appears in Rival [19]. Define $\mathscr{Q}(\mathbf{L})=\{[u, v]: u \leq$ $v$ and $\left.u \in J_{0}(\mathbf{L}), v \in M_{1}(\mathbf{L})\right\}$, where $M_{1}(\mathbf{L})$ denotes the set of meet irreducible elements of $\mathbf{L}$, including $1^{\mathrm{L}}$. If $\mathbf{L}$ is a finite distributive lattice, then the sublattices of $\mathrm{L}$ are precisely the subsets of $L$ having the form $K=L-\bigcup \mathscr{A}$, where $\mathscr{A} \subseteq \mathscr{Q}(\mathbf{L})$. In fact, the dual isomorphism for Sub $\mathbf{L}$ arises from the Galois connection between $L$ and $\mathscr{Q}(\mathbf{L})$ induced by the relation $\notin$, see also Vogt [22]. Although the dual isomorphisms in Rival [19] and the one presented here have some elements in common, there does not seem to be a trivial transition from one to the other.

\section{The proof of Theorem 2: Coverings in Sub L}

Proof. Let $\mathbf{L}$ be a finite distributive lattice, let $\mathbf{K} \in \operatorname{Sub} \mathbf{L}$, and let $x \in L-K$ with $\mathbf{K}^{*}=\operatorname{Sg}^{\mathbf{L}}(K \cup\{x\})$. There are four statements to prove:

(1) If $x=0^{\mathbf{K}^{*}}$, then $\mathbf{K}^{*}$ covers $\mathbf{K}$ and $\ell\left(\mathbf{K}^{*}\right)=1+\ell(\mathbf{K})$.

(2) If $x=1^{\mathbf{K}^{*}}$, then $\mathbf{K}^{*}$ covers $\mathbf{K}$ and $\ell\left(\mathbf{K}^{*}\right)=1+\ell(\mathbf{K})$.

(3) If $x$ splits a covering in $\mathbf{K}$, then $\mathbf{K}^{*}$ covers $\mathbf{K}$ and $\ell\left(\mathbf{K}^{*}\right)=1+\ell(\mathbf{K})$.

(4) If $x$ completes a square over $\mathbf{K}$, then $\ell\left(\mathbf{K}^{*}\right)=\ell(\mathbf{K})$. If $x$ completes a covering square over $K$, then $K^{*}$ covers $K$ as well.

If $x=0^{\mathbf{K}^{*}}$ or $x=1^{\mathbf{K}^{*}}$, then $\mathbf{K}^{*}$ covers $\mathbf{K}$ and $\ell\left(\mathbf{K}^{*}\right)=1+\ell(\mathbf{K})$, since $K^{*}=K \cup\{x\}$ in these cases. So (1) and (2) are clear.

To establish (3), let $x$ split a covering in $\mathbf{K}$. We want to prove that $\mathbf{K}^{*}$ covers $\mathbf{K}$ and that $\ell\left(\mathbf{K}^{*}\right)=1+\ell(\mathbf{K})$. 
We assume without loss of generality that $1^{\mathbf{L}} \in K$. Now suppose $b$ covers $a$ in $\mathbf{K}$ and that $x$ splits this covering. By distributivity, there is a unique join irreducible $p$ of $\mathbf{K}$ so that $p \leq b$ but $p \not \leq a$. Let $\varphi$ be $\Phi_{K}$ and define $\psi: J_{0}(\mathbf{L}) \rightarrow L$ via

$$
\psi(j)= \begin{cases}\varphi(j) \wedge x & \text { if } \varphi(j)=p \text { and } j \leq x \\ \varphi(j) & \text { otherwise }\end{cases}
$$

for all $j \in J_{0}(\mathbf{L})$.

LEMMA 2.1. $\psi=\Phi_{K^{*}}$

ProOF. By Theorem 3, we know $\Phi_{K^{*}}=\Phi_{K \cup\{x\}}$, since $K^{*}=\operatorname{Sg}^{\mathbf{L}} K \cup\{x\}$. This means $\Phi_{K^{*}}(j)=\bigwedge\{d: j \leq d$ and $d \in K \cup\{x\}\}$. So if $j \not \leq x$, then $\Phi_{K^{*}}(j)=\bigwedge\{d$ : $j \leq d \in K\}=\Phi_{K}(j)=\varphi(j)=\psi(j)$. So consider the case when $j \leq x$. In this case $\Phi_{K^{*}}(j)=x \wedge \wedge\{d: j \leq d \in K\}=x \wedge \varphi(j)$. Now if $j \leq a$ then $\varphi(j) \leq a$ since $a \in K$. Hence $\varphi(j) \neq p$ and so $\psi(j)=\varphi(j)=\varphi(j) \wedge x=\Phi_{K^{*}}(j)$. On the other hand, if $j \not \leq a$, then $\varphi(j)\lfloor a$. But $\varphi(j) \leq b$, since $j \leq x<b \in K$. By Theorem 3, $\varphi(j) \in J_{0}(\mathbf{K})$. This forces $\varphi(j)=p$. Therefore $\psi(j)=\varphi(j) \wedge x=\Phi_{K^{*}}$.

\section{LEMMA 2.2. Range $(\psi)=\operatorname{Range}(\varphi) \cup\{p \wedge x\}$.}

PROOF. To see this, we only need to find a join irreducible $j$ of $\mathbf{L}$ so that $\psi(j)=p$. Pick $j$ a join irreducible of $\mathbf{L}$ so that $j \leq b$ but $j \not \leq x$. Then $\varphi(j) \leq b$ and $\varphi(j) \not \leq a$. By Theorem 3, we know $\varphi(j)$ is a join irreducible in $\mathbf{K}$. Thus $\varphi(j)=p$. By the definition of $\psi$, we obtain $\psi(j)=\varphi(j)=p$. And in the proof of Lemma 2.1, we saw that $p \wedge x \in \operatorname{Range}(\psi)$.

From Theorem 3, $\Phi_{K^{*}}$ and $\Phi_{K}$ are distinct weakly independent maps. Since weakly independent maps with the same ranges must be the same, we conclude that $p \wedge x \notin$ $\operatorname{Range}(\varphi)$, in view of the Lemmas 2.1 and 2.2. That is $|\operatorname{Range}(\psi)|=1+|\operatorname{Range}(\varphi)|$. We also know that $1+\ell\left(\mathbf{K}^{*}\right)=|\operatorname{Range}(\psi)|$ and $1+\ell(\mathbf{K})=|\operatorname{Range}(\varphi)|$. Therefore, $\ell\left(\mathbf{K}^{*}\right)=1+\ell(\mathbf{K})$. In turn, this entails that $b$ covers $x$ in $\mathbf{K}^{*}$. Noting that $b=p \vee x$, we conclude that $p$ covers $p \wedge x$, since $[x, b]$ and $[p \wedge x, p]$ are isomorphic (transposed) intervals.

Now let $K \subseteq K^{\prime} \subseteq K^{*}$ where $K^{\prime} \in$ Sub L. Let $\tau=\Phi_{K^{\prime}}$. By Theorem 3, $\psi \leq \tau \leq \varphi$. According to the definition of $\psi$, if $\varphi(j) \neq p$ or $j \not \leq x$, then $\psi(j)=\tau(j)=\varphi(j)$. So consider any $j$, a join irreducible of $\mathbf{L}$ such that $\varphi(j)=p$ and $j \leq x$. For all such $j, p \wedge x=\psi(j) \leq \tau(j) \leq \varphi(j)=p$. So for each such $j$, either $\tau(j)=p \wedge x$ or $\tau(j)=p$. It follows that $\operatorname{Range}(\tau)=\operatorname{Range}(\psi)$ or $\operatorname{Range}(\tau)=\operatorname{Range}(\varphi)$. Since all three of these maps are weakly independent, we get $\tau=\psi$ or $\tau=\varphi$. Consequently, by Theorem 3, either $K^{\prime}=K^{*}$ or $K^{\prime}=K$. Therefore, $\mathbf{K}^{*}$ covers $\mathbf{K}$ in Sub $\mathbf{L}$. 
This concludes the proof of (3).

To establish (4), let $x$ complete a square over $\mathbf{K}$. We want to prove that $\ell\left(\mathbf{K}^{*}\right)=$ $\ell(\mathbf{K})$. Moreover, if $x$ completes a covering square over $\mathbf{K}$, then we want to prove that $\mathbf{K}^{*}$ covers $\mathbf{K}$ as well.

We assume without loss of generality that $1^{\mathbf{L}} \in K$. Again let $\varphi=\Phi_{K}$. Suppose $b>c>a$ and that $x \in K^{*}-K$ completes the square with $a, c, b$ over $\mathbf{K}$. Set $B=\left\{p \in J_{0}(\mathbf{L}): p \leq b\right.$ and $\left.p \not \leq c\right\}$. Define $\psi: J_{0}(\mathbf{L}) \rightarrow L$ so that

$$
\psi(j)= \begin{cases}\varphi(j) \wedge x & \text { if } \varphi(j) \in B \\ \varphi(j) & \text { otherwise }\end{cases}
$$

for all join irreducibles $j$ of $\mathbf{L}$. Lemma 2.3 below plays the same role in establishing (4) that Lemma 2.1 played in establishing (3).

LEMMA 2.3. $\psi=\Phi_{K^{*}}$

ProOF. By Theorem 3, we know $\Phi_{K^{*}}=\Phi_{K \cup\{x\}}$, since $K^{*}=\mathrm{Sg}^{\mathrm{L}} K \cup\{x\}$. This means $\Phi_{K^{*}}(j)=\bigwedge\{d: j \leq d$ and $d \in K \cup\{x\}\}$. So if $j \not \leq b$, then $\varphi(j) \not \leq b$ and $j \not \leq x$. This means $\Phi_{K^{*}}(j)=\bigwedge\{d: j \leq d \in K\}=\Phi_{K}(j)=\varphi(j)=\psi(j)$. So consider the case when $j \leq b$. In this case $\Phi_{K^{*}}(j)=x \wedge \wedge\{d: j \leq d \in K\}=$ $x \wedge \varphi(j)$. Now consider the subcase when $j \leq a$. Then $\varphi(j) \leq a=c \wedge x$, so $\varphi(j) \wedge x=\varphi(j)$. Therefore, $\Phi_{K^{*}}(j)=\psi(j)$. Thus, from this point on, we assume $j \leq b$ and $j \not \leq a$. Now if $j \leq c$, then $\varphi(j) \leq c$ (so $\varphi(j) \notin B$ ) and $j \not \leq x$, since $a=c \wedge x$. All this means that $\psi(j)=\varphi(j)=\Phi_{K^{*}}$. On the other hand, if $j \not c c$, then $j \leq x$, since $j \leq b=c \vee x$ and $j$ is join prime by distributivity. Under this hypothesis we also have $\varphi(j) \not \subset c$ while $\varphi(j) \leq b$. Thus $\varphi(j) \in B$. Therefore, $\psi(j)=\varphi(j) \wedge x=\Phi_{K^{*}}(j)$.

From the definition of $\psi$ it is evident that $|\operatorname{Range}(\psi)| \leq|\operatorname{Range}(\varphi)|$. So elementary facts about weakly independent maps entail that $\ell\left(\mathbf{K}^{*}\right) \leq \ell(\mathbf{K})$. On the other hand, $K \subseteq K^{*}$ implies that $\ell\left(\mathbf{K}^{*}\right) \geq \ell(\mathbf{K})$. Therefore, $\ell\left(\mathbf{K}^{*}\right)=\ell(\mathbf{K})$.

Now assume, in addition that $b$ covers $c$ covers $a$ in $\mathbf{K}$ so that $x$ completes a covering square with $a, c, b$ over $\mathbf{K}$. Then $B$ has exactly one element. Call it $p$. Then $\operatorname{Range}(\psi)=(\operatorname{Range}(\varphi)-\{p\}) \cup\{p \wedge x\}$. By Theorem 3 we know that $\psi<\varphi$ in $\mathscr{W}_{\mathbf{L}}$. Since these maps are weakly independent, they must have different ranges. In particular, $p \wedge x \neq p$. Thus, we know that $p \not x$. Since the lengths of $\mathbf{K}$ and $\mathbf{K}^{*}$ are the same, we know that $b$ covers $x$ covers $a$. Therefore, $p \vee x=b$. This entails that $p$ covers $p \wedge x$ since the transposed intervals $[x, b]$ and $[p \wedge x, p]$ are isomorphic.

Finally, suppose $K^{\prime} \in \operatorname{Sub} \mathbf{L}$ with $K \subseteq K^{\prime} \subseteq K^{*}$ and let $\tau=\Phi_{K^{\prime}}$. By Theorem 3, we know that $\psi \leq \tau \leq \varphi$ in $\mathscr{W}_{\mathbf{L}}$. Let $j$ be a join irreducible element of $\mathbf{L}$. If $\varphi(j) \neq p$, then $\psi(j)=\tau(j)=\varphi(j)$. If $\varphi(j)=p$, then $\tau(j) \in\{p \wedge x, p\}$ since $p$ 
covers $p \wedge x$ in $\mathbf{K}^{*}$ and $\tau(j)$ must lie in the interval in $\mathbf{K}^{*}$ between $\psi(j)=p \wedge x$ and $\varphi(j)=p$. This means that either $\operatorname{Range}(\tau)=\operatorname{Range}(\psi)$ or $\operatorname{Range}(\tau)=\operatorname{Range}(\varphi)$ or $\operatorname{Range}(\tau)=\operatorname{Range}(\psi) \cup \operatorname{Range}(\varphi)$. But the latter alternative is not possible, since $|\operatorname{Range}(\psi)|=|\operatorname{Range}(\tau)|=|\operatorname{Range}(\varphi)|$ because $\ell\left(\mathbf{K}^{*}\right)=\ell\left(\mathbf{K}^{\prime}\right)=\ell(\mathbf{K})$ and the maps are weakly independent. So once again we can conclude that $\tau=\psi$ or $\tau=\varphi$. By Theorem 3, we conclude that $\mathbf{K}^{\prime}=\mathbf{K}^{*}$ or $\mathbf{K}^{\prime}=\mathbf{K}$. Consequently, $\mathbf{K}^{*}$ covers $\mathbf{K}$ in Sub L.

\section{A transposition principle}

While we know that $\operatorname{Sub} \mathbf{L}$ fails to be a modular lattice whenever $\mathbf{L}$ is not a chain, it is nevertheless true that some instances of Dedekind's Transposition Principle hold in $\operatorname{Sub} \mathbf{L}$ as long as $\mathbf{L}$ is a finite distributive lattice. This is the content of the next theorem.

THEOREM 4. Let $\mathbf{L}$ be a finite distributive lattice, let $\mathbf{K}$ be a sublattice of $\mathbf{L}$, and let $a, b \in K$ with $a<b$. Define $K_{0}, K_{1} \in \operatorname{Sub} \mathbf{L}$ by $K_{0}=K \wedge[a, b]$ and $K_{1}=K \vee[a, b]$, where $[a, b]$ denotes the interval in $\mathbf{L}$ from $a$ to $b$. Then the maps $\Upsilon:\left[K_{0},[a, b]\right] \rightarrow$ $\left[K, K_{1}\right]$ and $\Delta:\left[K, K_{1}\right] \rightarrow\left[K_{0},[a, b]\right]$, defined so that

$$
\Upsilon(M)=M \vee K \quad \Delta(N)=N \wedge[a, b]
$$

for all $M \in\left[K_{0},[a, b]\right]$ and all $N \in\left[K, K_{1}\right]$, are inverses of each other, establishing an isomorphism between the transposed intervals $\left[K_{0},[a, b]\right]$ and $\left[K, K_{1}\right]$ in Sub $\mathbf{L}$.

PROOF. Both $\Upsilon$ and $\Delta$ are order preserving maps between two finite lattices. Therefore, the two lemmas below suffice to establish the theorem.

LEMMA 4.1. $\Delta(\Upsilon(M))=M$ for all $M \in \operatorname{SubL}$ with $K \cap[a, b] \subseteq M \subseteq[a, b]$.

PROOF. It is evident that $M \subseteq \Delta(\Upsilon(M))$. To prove the reverse inclusion, let $y \in \Delta(\Upsilon(M))$. Our goal is to show that $y \in M$. Now $\Upsilon(M)$ is the subuniverse $N$ of L generated by $M \cup K$ and $\Delta(N)$ is $N \cap[a, b]$. By distributivity, it follows that there is a natural number $n$ and elements $x_{i} \in M$ and $z_{i} \in K$ for all $i<n$ such that

$$
a \leq y=\bigvee_{i<n}\left(x_{i} \wedge z_{i}\right) \leq b
$$

But now we can write

$$
y=(y \vee a) \wedge b=\bigvee_{i<n}\left(\left(\left(x_{i} \vee a\right) \wedge b\right) \wedge\left(\left(z_{i} \vee a\right) \wedge b\right)\right)=\bigvee_{i<n}\left(x_{i} \wedge\left(\left(z_{i} \vee a\right) \wedge b\right)\right.
$$

Since $\left(z_{i} \vee a\right) \wedge b \in K_{0} \subseteq M$ for each $i<n$, we conclude that $y \in M$, as desired. 
LEMMA 4.2. $\Upsilon$ maps $\left[K_{0},[a, b]\right]$ onto $\left[K, K_{1}\right]$.

Proof. Let $N \in \operatorname{Sub} \mathbf{L}$ with $K \subseteq N \subseteq K_{1}$. First, we suppose that $N$ covers $K$ in Sub $\mathbf{L}$. Since $0^{\mathbf{K}}=0^{\mathbf{K}_{1}}$ and $1^{\mathbf{K}}=1^{\mathbf{K}_{1}}$, we can invoke either part (3) or part (4) of Theorem 1 to obtain an element $y \in N-K$ and elements $u, v \in K$ with $u<y<v$ in $\mathbf{L}$ so that $N=\mathrm{Sg}^{\mathbf{L}}(K \cup\{y\})$. From the length considerations in parts (3) and (4) of Theorem 2, it follows that $v$ covers $y$ covers $u$ in $\mathbf{N}$.

Now let $M=K_{0} \vee\{(y \vee a) \wedge b\}$. What we want is $(y \vee a) \wedge b \in N-K$, because then $Y=M \vee K=\Upsilon(M)$, establishing that $\Upsilon$ maps onto $\left[K, K_{1}\right]$ (in the case that $N$ covers $K$ ).

Next, we eliminate the case when $y \vee a=v \vee a$. In this case, we would have $y \vee x=v \vee x$ for all $x \in[a, b]$. Since $y \in Y \subseteq K \vee[a, b]$, using distributivity we can decompose $y$ as

$$
y=z \vee \bigvee_{i<n}\left(x_{i} \wedge z_{i}\right)
$$

where $z \in K, x_{i} \in[a, b]$, and $z_{i} \in K$. With this decomposition we also have

$$
x_{i} \wedge z_{i} \leq\left(y \vee x_{i}\right) \wedge z_{i} \leq\left(y \vee x_{i}\right) \wedge\left(y \vee z_{i}\right) \leq y \vee\left(x_{i} \wedge z_{i}\right) \leq y .
$$

This means we can rewrite our decomposition to get

$$
y=z \vee \bigvee_{i<n}\left(x_{i} \wedge z_{i}\right)=z \vee \bigvee_{i<n}\left(\left(y \vee x_{i}\right) \wedge z_{i}\right)=z \vee \bigvee_{i<n}\left(\left(v \vee x_{i}\right) \wedge z_{i}\right)
$$

But $y \leq v$, therefore

$$
\begin{aligned}
y=y \wedge v & =\left(z \vee \bigvee_{i<n}\left(\left(v \vee x_{i}\right) \wedge z_{i}\right)\right) \wedge v \\
& =(z \wedge v) \vee \bigvee_{i<n}\left(\left(v \vee x_{i}\right) \wedge v \wedge z_{i}\right)=(z \wedge v) \vee \bigvee_{i<n}\left(v \wedge z_{i}\right)
\end{aligned}
$$

However, this means that $y \in K$, contradicting our choice of $y$ and so eliminating the case when $y \vee a=v \vee a$.

Consider the case when $y \vee a<v \vee a$. Since $v$ covers $y$ in $N$, it follows that $(y \vee a) \wedge v=y$; but also $(y \vee a) \vee v=y \vee v$. Hence, the transposed intervals $[y, v]$ and $[y \vee a, v \vee a$ ] of $\mathbf{N}$ are isomorphic. Since $v \in K$ and $y \in N-K$, we obtain $y \vee a \in N-K$. It also follows that $v \vee a$ covers $y \vee a$ in $\mathbf{N}$. But now $u \vee a \in K$ so $y \vee a \neq u \vee a$. This entails that $y \vee a$ covers $u \vee a$ in $\mathbf{N}$. So from $v$ covers $y$ covers $u$ in $\mathbf{N}$ we have concluded that $v \vee a$ covers $y \vee a$ covers $u \vee a$ in $\mathbf{N}$ and $y \vee a \in N-K$. Now reasoning in a dual fashion (replacing $a$ with $b$ and $\vee$ with $\wedge$ ), 
we can further conclude that $(v \vee a) \wedge b$ covers $(y \vee a) \wedge b$ covers $(u \vee a) \wedge b$ in $\mathbf{N}$ and $(y \vee a) \wedge b \in N-K$. Thus, we are finished in the case when $N$ covers $K$.

Now assume that $N \in \operatorname{Sub} \mathbf{L}$ with $K \subseteq N \subseteq K_{1}$ and that $N^{\prime}$ belongs to the range of $\Upsilon$ whenever $N^{\prime} \in$ Sub L with $K \subseteq N^{\prime}$ and $N^{\prime}$ is a proper subset of $N$. Pick $N_{*} \in$ Sub L with $K \subseteq N_{*}$ so that $N$ covers $N_{*}$. Pick $M_{*} \in\left[K_{0},[a, b]\right]$ such that $K \vee M_{*}=N_{*}$. By the part of the argument for this lemma that we completed above, there is $M \in \operatorname{Sub} \mathbf{L}$ with $M_{\star} \subseteq M \subseteq[a, b]$ so that $\Upsilon(M)=K \vee M=N_{*} \vee M=N$. This means $\Upsilon$ is onto.

Having established Lemmas 4.1 and 4.2 , the proof of Theorem 4 is complete.

\section{A comparison of characterizations}

As mentioned in the Introduction, a series of investigations begun by Hashimoto [11] leads to a characterization of maximal sublattices of a finite distributive lattice which differs from the one we gave in Theorems 1 and 2. In addition to Hashimoto's paper, those of Chen, Koh and Tan [8], Rival [18], Tůma [21], and Adams, Dwinger and Schmid [3] provide the clearest view of the characterization. In order to make comparison with our approach easy, we state a version of this characterization next, following the presentation in [3] closely.

Given a finite distributive lattice $\mathbf{L}$ and a maximal proper sublattice $\mathbf{S}$ there must be a join irreducible element $j$ of $\mathbf{L}$ which is omitted from $\mathbf{S}$. Indeed, as a consequence of distributivity, this element is easily seen to be unique. The characterizations in the style of the work begun by Hashimoto describe which join irreducible elements can be omitted and which maximal sublattices omit them. It is worth noting that the omitted join irreducible $j$ may not uniquely determine the omitting maximal sublattice S. Examination of the maximal sublattices of the cube $2^{3}$ reveals this. A pair $(j, k)$ of join irreducible elements of $\mathbf{L}$ is said to be critical (for example in [3]) provided $k \not z j$ and for every $l \in J_{0}(\mathbf{L})$

$$
j<l \Rightarrow k \leq l \text { and } l<k \Rightarrow l \leq j .
$$

For a critical pair $(j, k)$ we define

$$
S_{j, k}=\{x \in L: j \leq x \Rightarrow k \leq x\} .
$$

Since every join irreducible element is also join prime, it follows that $S_{j . k} \in \operatorname{Sub} \mathbf{L}$. (It should be noted that while $j \notin S_{j . k}$, it is true that $x \in S_{j . k}$ for all $x \geq k$. So while $j$ is omitted, $k$ is not.) Finally, in case $1^{\mathrm{L}}$ is join irreducible, we let

$$
S_{1}=\left\{x \in L: x<1^{\mathrm{L}}\right\} .
$$


It is clear that if $\left(0^{\mathbf{L}}, k\right)$ is critical, then $k$ must be the unique member of $L$ covering $0^{\mathrm{L}}$ and

$$
S_{0 . k}=\left\{x \in L: 0^{\mathbf{L}}<x\right\} .
$$

Here is our reformulation of the characterization theorem underlying the line of research cited above.

THEOREM 5. Let $\mathbf{L}$ be a finite distributive lattice. The maximal proper subuniverses of $\mathbf{L}$ are exactly the subuniverses $S_{j . k}$ where $(j, k)$ is a critical pair of join irreducible elements of $\mathbf{L}$ and the subuniverse $S_{1}$, in case $1^{\mathbf{L}}$ is join irreducible.

Proofs of this theorem (or one of its variants) can be found either explicitly or implicitly in several of the papers cited in the Introduction. We devised this formulation on the basis of Tủma's paper [21]. The proof implicit in Tủma's paper has two steps. First, Tưma notes that the listed subuniverses constitute an antichain, and then argues that any subuniverse extends to one of those listed. Tủma's arguments, like Hashimoto's, are framed in the language of order ideals. These arguments can all be converted, in the context of finite distributive lattices, more or less routinely into arguments about join irreducible elements, in view of the duality observed by Birkhoff [6] in 1933 between finite distributive lattices and the order ideals of their join irreducible elements.

Our characterization, given in Theorems 1 and 2, concerns adding elements to a sublattice $\mathbf{S}$ to obtain a sublattice $\mathbf{S}^{*}$ which covers $\mathbf{S}$. On the other hand, the characterization given in Theorem 5 concerns omitting a join irreducible element from a sublattice $\mathbf{S}$ to obtain a sublattice $\mathbf{S}_{*}$ which is covered by $\mathbf{S}$. These approaches complement each other. Here we formulate Theorem 6. a more explicit version of Theorem 5 revealing how the omitted join irreducible elements fit with the maximal sublattices, and prove it on the basis of Theorems 1 and 2. (Alternatively, this theorem follows easily from Theorems 1, 2, and 5.)

In a finite lattice, the nonzero join irreducibles are those elements that cover exactly one other element. As a matter of notation, if $j$ is a nonzero join irreducible element we use $j_{*}$ to denote the unique element covered by $j$.

THEOREM 6. Let $\mathbf{L}$ be a finite distributive lattice. Then

(1) If $1^{\mathrm{L}}$ is join irreducible in $\mathbf{L}$, then $S_{1}$ is maximal.

(2) If $0^{\mathbf{L}}$ is covered by a unique element $k$ in $\mathbf{L}$, then $\left(0^{\mathbf{L}}, k\right)$ is critical and $S_{0 . k}$ is maximal.

(3) If $(j, k)$ is critical and $j<k$, then $k$ covers $j_{*}$ in $S_{j . k}$ (and $j$ splits this covering). Hence $S_{j . k}$ is maximal.

(4) If $(j, k)$ is critical and $j \nless k$, then $j$ completes the covering square in $S_{j . k}$ with remaining members $j_{*}, j_{*} \vee k$, and $j \vee k$. Hence $S_{j . k}$ is maximal. 
Conversely, let $S$ be a maximal proper subuniverse of $\mathbf{L}$ and let $j$ be the unique join irreducible of $\mathbf{L}$ not in $S$. Then one of the following cases happens:

(1') $1^{\mathbf{L}}$ is join irreducible in $\mathbf{L}$ and $S=S_{1}$.

$\left(2^{\prime}\right) \quad k$ is the unique element covering $0^{\mathrm{L}}$ in $\mathbf{L}$ and $S=S_{0, k}$.

(3') There is a join irreducible $k>j$ such that $(j, k)$ is critical, $k$ covers $j_{*}$ in $S$ (and $j$ splits this covering), and $S=S_{j, k}$.

(4') There is a join irreducible $k$ incomparable to $j$ such that $(j, k)$ is critical, $j_{*} \prec j_{*} \vee k \prec j \vee k$ in $\mathbf{S}$, and $S=S_{j . k}$.

PROOF. Items (1) and (2) are immediate.

Consider (3). Let $(j, k)$ be a critical pair with $j<k$. To see that $k$ covers $j_{*}$ in $\mathbf{S}_{j . k}$ suppose to the contrary that we have $s \in S_{j . k}$ with $j_{*}<s<k$. Then there must be an element $l$ join irreducible in $\mathbf{L}$ so that $j_{*}<j_{*} \vee l \leq s<k$. Because $(j, k)$ is critical we find $l \leq j$. In turn, this gives us $l=j$ and $j<s<k$. But likewise, this means there is $m$ join irreducible in $\mathbf{L}$ so that $j<j \vee m \leq s<k$. Again, since $(j, k)$ is critical and $m<k$, we find that $m \leq j$, producing the absurdity $j<j$. Therefore $j$ splits the covering $j_{*} \prec k$ in $\mathbf{S}_{j . k}$. Now observe that every join irreducible element of $\mathbf{L}$ other than $j$ belongs to $S_{j . k}$. Consequently, $\operatorname{Sg}^{\mathrm{L}}\left(S_{j . k} \cup\{j\}\right)=L$. Hence $S_{j . k}$ is maximal by Theorem 2 .

Now consider (4). Let $(j, k)$ be a critical pair with $j$ and $k$ incomparable. Because $j$ and $k$ are incomparable, we know that $j \wedge k \leq j_{*}$. This entails that the $\mathbf{L}$-intervals $\left[j_{*}, j\right]$ and $\left[j_{*} \vee k, j \vee k\right]$ are transposed, as are $\left[j_{*}, j_{*} \vee k\right]$ and $[j, j \vee k]$. Since we already know that $j$ covers $j_{*}$, it suffices to show that $j_{*} \vee k$ covers $j_{*}$ in L. Suppose to the contrary that $j_{*}<x<j_{*} \vee k$. Then there must be $l$ join irreducible in $\mathbf{L}$ so that $j_{*}<j_{*} \vee l \leq x<j_{*} \vee k$. Because $(j, k)$ is critical, we know that $l \leq j$. Now $j=l<k$ is impossible and $l \leq j_{*}<j_{*} \vee l$ is impossible, which is absurd. Hence $j$ completes a covering square in $S_{j . k}$ (and as before $L=\operatorname{Sg}^{\mathrm{L}}\left(S_{j . k} \cup\{j\}\right)$ ). Therefore, $S_{j . k}$ is maximal by Theorem 2 .

For the converse, we suppose that $S$ is a maximal subuniverse of $\mathbf{L}$ and that $j$ is the unique join irreducible of $\mathbf{L}$ which is not in $S$. We suppose that neither Case $1^{\prime}$ nor Case $2^{\prime}$ holds. Thus $0^{\mathbf{L}}<j<1^{\mathbf{L}}$, and $0^{\mathbf{L}}, 1^{\mathbf{L}} \in S$. Pick $a, b \in S$ so that $\mathbf{S}$-interval $[a, b]$ is as short as possible subject to the condition that $a<j<b$. This means that $a=j_{*}$, the unique element of $L$ covered by $j$, since every join irreducible of $\mathbf{L}$ properly smaller than $j$ belongs to $S$. This also means that $b$ is the meet of all elements of $S$ which are larger than $j$. As a consequence, if $s \in S$ and $j \leq s$, then $b \leq s$.

Now notice that since $j<b$ there must be a join irreducible $k$ of $\mathbf{L}$ such that $k \leq j$ and $k \leq b$. Let $k$ be selected which is minimal in $\mathbf{L}$ subject to these constraints.

LEMMA 6.1. $(j, k)$ is critical. 
PROOF. Let $l$ be a join irreducible of $\mathbf{L}$. First suppose $k<l$. Then $l \in S$, and consequently $b \leq l$. Next suppose $l<k$. By the minimality of $k$, we have $l \leq j$.

\section{LEMMA 6.2. $S=S_{j . k}$.}

PROOF. By the maximality of $S$ it is enough to prove that $S \subseteq S_{j . k}$. So let $s \in S$. Suppose $j \leq s$. But then $k \leq b \leq s$. This means $s \in S_{j . k}$. Therefore, $S \subseteq S_{j . k}$.

CASE I: $b$ covers $a$ in $\mathbf{S}$.

Since $k \not \leq j$, we see that $a<a \vee k$. Since $k \leq b$, we get $a \vee k \leq b$. Since $k \in S$ and $b$ covers $a$ in $S$, we conclude that $a \vee k=b$. Hence $j<a \vee k$. But $j$ is join prime and $a<j$. Thus, $j \leq k$. This entails that $b \leq k$. Consequently, $b=k$ and so $k=b$ covers $j_{*}=a$ in $S$. Therefore, Case $3^{\prime}$ holds.

CASE II: The $\mathbf{S}$-interval $[a, b]$ has at least three elements.

Pick $c \in S$ so that $c<b$ and $c$ covers $a$ in $S$. It does no harm to assume that $k$ satisfies the additional constraint that $k \leq c$. It follows that $a \vee k=c$ (by reasoning as in Case I). Now notice that every join irreducible of $\mathbf{L}$ which is properly below $k$ must also be below $j$ and below $j_{*}=a$. This entails that $j \wedge k=j_{*} \wedge k=a \wedge k=k_{*}$. Thus the intervals $\left[j_{*}, j_{*} \vee k\right]=[a, c]$ and $\left[j_{*} \wedge k, k\right]=\left[k_{*}, k\right]$ are transposed. Since $k$ covers $k_{*}$ in $\mathbf{L}$, we discover that $c=j_{*} \vee k$ covers $a=j_{*}$ in $\mathbf{L}$. Again, invoking the Transposition Principle, for $\left[j_{*}, j\right]$ and $\left[j_{*} \vee k, j \vee k\right]$, we see that $j \vee k$ covers $j_{*} \vee k$ in $\mathbf{L}$. (Also, $j \vee k$ covers $j$ since $j_{*} \vee k$ covers $j_{*}$ ). So Case 4 ' will be established once we prove that $j \vee k=b$, since $b \in S$.

Now if $j \vee k \in S$, then we know that $j \vee k=b$. So suppose that $j \vee k \notin S$. Since $S$ is a maximal subuniverse, this means that $j$ can be written as a join of meets of members of $S \cup\{j \vee k\}$. Since $j$ is join irreducible it must actually be a meet of members of $S \cup\{j \vee k\}$. Since $S$ is closed under forming meets, it follows that $j$ must have one of the following forms: $j \vee k, s$, or $s \wedge(j \vee k)$ where $s \in S$. It follows that $j=s \wedge(j \vee k)$ for some $s \in S$. As $j<b$, we can further suppose that $s \leq b$. But because $j<s$ implies $b \leq s$, we conclude that $j=b \wedge(j \vee k)=(b \wedge j) \vee(b \wedge k)=j \vee k$ which is impossible. This completes the demonstration of Case $4^{\prime}$.

We conclude this section with a proof of a variant of Theorem 2 based on Theorem 5 .

TheOREM 7. Let $\mathbf{K} \in \operatorname{Sub} \mathbf{L}$, and let $x \in L-K$ with $\mathbf{L}=\operatorname{Sg}^{\mathbf{L}}(K \cup\{x\})$.

(1) If $x=0^{\mathbf{L}}$, then $\mathbf{L}$ covers $\mathbf{K}$ and $\ell(\mathbf{L})=1+\ell(\mathbf{K})$.

(2) If $x=1^{\mathbf{L}}$, then $\mathbf{L}$ covers $\mathbf{K}$ and $\ell(\mathbf{L})=1+\ell(\mathbf{K})$.

(3) If $x$ splits a covering in $\mathbf{K}$, then $\mathbf{L}$ covers $\mathbf{K}$ and $\ell(\mathbf{L})=1+\ell(\mathbf{K})$.

(4) If $x$ completes a covering square over $\mathbf{K}$, then $\mathbf{L}$ covers $\mathbf{K}$ and $\ell(\mathbf{L})=\ell(\mathbf{K})$.

PROOF. Statements (1) and (2) are immediate. 
Since $\operatorname{Sg}^{\mathbf{L}}(K \cup\{x\})=L$ and since $\mathbf{L}$ is distributive, every element of $L$ can be expressed as a join of meets finite subsets of $K \cup\{x\}$. In particular, a join irreducible element $l$ of $\mathbf{L}$ must be of one of these three forms: $l=x$ or $l \in K$ or $l=x \wedge d$ for some $d \in K$ with $d \leq b$. As a special case, if $l$ is a join irreducible of $\mathbf{L}$ and $x<l$, then $l \in K$.

Now, statements (3) and (4) concern an interval $[a . b]$ where $a, b \in K$. In statement (3) we have that $b$ covers $a$ in $\mathbf{K}$, while in statement (4) we have a third element $c \in K$ so that $x$ completes a covering square over $K$ with the other three points being $a, c$, and $b$. Fix these elements of $K$.

LEMMA 7.1. If $a \leq e \leq b$ and $e \in K$, then either $x \wedge e=x$ or $x \wedge e=a$.

Proof. In case $b$ covers $a$ in $K$, we have $e=b$ or $e=a$. So the lemma follows.

So suppose $a \prec c \prec b$ in $\mathbf{K}$ and $x$ completes the square. So $[a, x]$ and $[c, b]$ are transposed intervals. Now $(x \wedge e) \in[a, x]$. By the Transposition Principle, $x \wedge e=$ $((x \wedge e) \vee c) \wedge x$. But then $x \wedge e=(x \vee c) \wedge(e \vee c) \wedge x=b \wedge(e \vee c) \wedge x=(e \vee c) \wedge x$. Now $e \vee c$ is an element of $K$ between $c$ and $b$. Because $b$ covers $c$ in $\mathbf{K}$, we have that

$$
x \wedge e= \begin{cases}b \wedge x=x & \text { if } e \vee c=b \\ c \wedge x=a & \text { if } e \vee c=c .\end{cases}
$$

CASE I: $x$ is a join irreducible of $\mathbf{L}$.

Select a join irreducible $k$ of $\mathbf{L}$ minimal with respect to the constraints that $k \not x x$ and $k \leq b$ (and for statement $(4) k \leq c$ ).

\section{LEMMA 7.2. Every join irreducible of $\mathbf{L}$, other than $x$, belongs to $K$.}

PROOF. Suppose that $l$ is a join irreducible of $\mathbf{L}$. We know that $l=x$ or $l \in K$ or $l=x \wedge d$ for some $d \in K$ with $d \leq b$. We only need the examine the last case. For this, observe $a \vee l=a \vee(x \wedge d)=x \wedge(a \vee d)$. Since $a \leq a \vee d \leq b$, Lemma 7.1 applies with $e=a \vee d$. So either $a \vee l=x$ or $a \vee l=a$. Under the first alternative, we conclude that $l=x$, because $x$ is join irreducible and $a<x$. Under the second alternative, we have $l \leq a \wedge d \leq x \wedge d=l$. As a consequence, $l=a \wedge d \in K$.

LEMMA 7.3. $x \vee k=b$.

ProOF. Since $k$ is a join irreducible other than $x$, we know that $k \in K$. Also $a<a \vee k \leq b$. In case $b$ covers $a$, we get that $a \vee k=b$, and hence $x \vee k=b$. In case $a \prec c \prec b$ in $\mathbf{K}$ and $x$ completes the square, we have $a<a \vee k \leq c$. So, $a \vee k=c$. Since $[a, c]$ and $[x, b]$ are transposed intervals, $x \vee k=b$. 
LEMMA 7.4. $(x, k)$ is critical.

PROOF. Let $l$ be a join irreducible of $\mathbf{L}$. First suppose $l<k$. Then $l \leq x$, by the minimality of $k$. Now suppose $x<l$. We know that $l \in K$ and that $x \leq l \wedge b$. In case $b$ covers $a$ in $\mathbf{K}$, we conclude that $l \wedge b=b$, and hence $k \leq b \leq l$. In case $x$ completes the covering square, we know that the only element of $[x, b]$ which belongs to $K$ is $b$, since this interval transposes down to $[a, c]$ and $c$ covers $a$ in $\mathbf{K}$. So again, $k \leq b=l \wedge b \leq l$.

\section{LEMMA 7.5. $S_{x \cdot k} \subseteq K$.}

PROOF. Suppose $s \in S_{x \cdot k}$. Then either $x \not \leq s$ or $k \leq s$. If $x \not \leq s$, then $x$ is not among the join irreducibles of $\mathbf{L}$ which are less than or equal to $s$. This means all those join irreducibles belong to $K$. Since $s$ is just the join of all those join irreducibles, we conclude that $s \in K$. So suppose that $x \leq s$. Then $k \leq s$ as well. So $x \vee k \leq s$. Since $x \vee k=b \in K$, this means that in any representation of $s$ as a join of join irreducibles. any occurrence of $x$ can be replaced by $b$, resulting in a representation of $s$ as a join of elements of $K$. Hence $s \in K$. Consequently, $S_{x . k} \subseteq K$.

Now Theorem 5 tells us that $S_{x \cdot k}$ is a maximal subuniverse of $\mathbf{L}$. Hence $S_{x \cdot k}=K$, and $K$ is a maximal subuniverse of $\mathbf{L}$. To conclude the proof in Case $\mathrm{I}$, we have to establish the statements about length. For this purpose it is enough to count the join irreducible elements of $\mathbf{K}$. Of course, every element of $K$ which is a join irreducible of $\mathbf{L}$ is also a join irreducible of $\mathbf{K}$.

LEMMA 7.6. Ifl is a join irreducible of $\mathbf{K}$, then l is a join irreducible of $\mathbf{L}$ or $l=b$; moreover, $b$ is a join irreducible of $\mathbf{K}$.

PROOF. Suppose that $l$ is a join irreducible of $\mathbf{K}$ that is not a join irreducible of $\mathbf{L}$. Now $l$ can be expressed as a join of elements which are join irreducibles on $\mathbf{L}$. The element $x$ must be among those join irreducibles, since the remaining join irreducibles of $\mathbf{L}$ all belong to $K$. Indeed, $l=x \vee e$ where $e \in K$ since it is the join of the other join irreducibles. Hence, $x<l$. This means $b \leq l$ and consequently, $l=b \vee e$. It follows that $l=b$, since $l$ is a join irreducible of $\mathbf{K}$. Were $b$ join reducible in $\mathbf{K}$, then every join irreducible of $\mathbf{K}$ would be a join irreducible of $\mathbf{L}$ and $b$ could be expressed as a join of elements, each a join irreducible of $\mathbf{L}$ different from $x$. However, since $b=x \vee k$ is already an irredundant join of elements join irreducible in $\mathbf{L}$, the uniqueness of such decompositions would entail $x \in K$, which is impossible.

Now in the case that $b$ covers $a$ in $\mathbf{K}$, we saw (in the proof of Lemma 7.3) that $x<b=a \vee k$. Since $x$ is join prime but $a<x$, we deduce $x<k$. This means that $k=a \vee k=b$. Consequently, $b$ is a join irreducible of $\mathbf{L}$. This implies that 
$\ell(\mathbf{L})=\ell(\mathbf{K})+1$. In the case that $a<c<b$ in $\mathbf{K}$ and $x$ completes this square, we see that while $b$ is join irreducible in $\mathbf{K}$, it is join reducible as $b=x \vee c$ in $\mathbf{L}$. This implies that $\ell(\mathbf{L})=\ell(\mathbf{K})$.

CASE II: $x$ is not a join irreducible of $\mathbf{L}$.

Pick a $j$ a join irreducible of $\mathbf{L}$ so that $a<a \vee j \leq x$. We can also pick $d \in K$ with $d \leq b$ so that $j=x \wedge d$.

LEMMA 7.7. The intervals $[a \wedge d, d]$ and $[a, b]$ are transposed.

PROOF. We need only show that $b=a \vee d$. We know that either $x \wedge(a \vee d)=x$ or $x \wedge(a \vee d)=a$. Since the latter alternative leads to $a \vee j=a$ we must reject it. Hence $x=x \wedge(a \vee d)<a \vee d \leq b$. In case $b$ covers $a$, we conclude that $a \vee d=b$. In case $x$ completes the covering square, we know once more that $a \vee d=b$, since $[x, b]$ transposes down to $[a, c]$ and $c$ covers $a$ in $\mathbf{K}$.

\section{LEMMA 7.8. $K \cup\{j\}$ generates $L$.}

ProOf. Since $[a \wedge d, d]$ and $[a, b]$ are transposed intervals, the perspectivity maps ensure that $j=x \wedge d$ and $x=j \vee a$. Since $K \cup\{x\}$ generates $L$. we have that $K \cup\{j\}$ also generates $L$ (and conversely).

These last two lemmas mean that Case II reduces to Case I. This finishes our proof of Theorem 7.

\section{Remarks}

The concept of a weakly independent map was motivated by the work of Czédli. Huhn and Schmidt [9]. They call a set $H$ of elements of a lattice $\mathbf{L}$ weakly independent provided $h \leq h_{0} \vee \cdots \vee h_{n-1}$ implies that $h \leq h_{i}$ for some $i<n$, for all h. $h_{0} \ldots \ldots h_{n-1} \in H$. In [9] Czédli, Huhn and Schmidt prove that if $H$ is a maximal weakly independent subset of a finite distributive lattice $\mathbf{L}$, then $|H|=\left|J_{0}(\mathbf{L})\right|$. To establish this theorem, they defined $\varphi_{H}: J_{0}(\mathbf{L}) \rightarrow L$ by $\varphi_{H}(j)=\bigwedge\{h \in H: j \leq h\}$, and showed that $\varphi_{H}$ is a one-to-one mapping onto $H$. We note that in a distributive lattice, the range of each weakly independent map is a weakly independent set in the sense of Czédli, Huhn and Schmidt.

There are simple examples showing that Theorem 3 fails for modular lattices. However, we still have the conclusion from the early lore of lattice theory that $\mathscr{S}_{\mathbf{L}}$ and $\mathscr{K}_{\mathbf{L}}$ are dually isomorphic for any finite lattice $\mathbf{L}$. In the absence of distributivity, the join irreducibles seem to exhibit a more complicated behavior with respect to topological closure operators. We do not know how far any of the results in Theorem 2 and 
Theorem 4 extend to finite modular lattices. The examples displayed in Figure 1 show that the hypothesis of distributivity in Theorem 2 cannot be completely abandoned. However, none of these examples is modular. The only negative fact for modular lattices we have is that the statement concerning the lengths of sublattices in Theorem 2 (3) fails: the length must increase by at least 2 if $\mathbf{K}$ is $\mathbf{M}_{\text {. }}$.

Finite distributive lattices are bounded in the sense of McKenzie [14]; they are, in some sense, the lattices encountered at the earliest stage in the recursive construction of the finite bounded lattices. Theorem 7 of [4] provides a useful condition necessary for any maximal sublattice of a finite bounded lattice. It is an intriguing problem to find sufficient conditions, and, indeed, to characterize the maximal sublattices of finite bounded lattices.

\section{References}

[1] M. Abad and M. E. Adams. 'The Frattini sublattice of a finite distributive lattice'. Algebra Universalis 32 (1994), 314-329.

[2] M. E. Adams. 'The Frattini sublattice of a distributive lattice', Algebra Universalis 3 (1973). 216-228.

[3] M. E. Adams. P. Dwinger and J. Schmid. 'Maximal sublattices of finite distributive lattices'. Algebra Universalis 36 (1996), 488-504.

[4] M. E. Adams, R. Freese, J. B. Nation and J. Schmid. "Maximal sublattices and Frattini sublattices of bounded lattices'. J. Austral. Math. Soc. (Series A) 63 (1997). 110-127.

[5] R. Balbes and P. Dwinger, Distributive Lattices (University of Missouri Press, Missouri. 1974).

[6] G. Birkhoff. 'On the combination of subalgebras', Proc. Cambridge Philos. Soc. 29 (1933). 441464.

[7] - Lattice Theor: Amer. Math. Soc. Colloq. Publ. 25, 3rd edition (Amer. Math. Soc.. Providence, RI, 1967).

[8] C. C. Chen, K. M. Koh and S. K. Tan. 'Frattini sublattices of distributive lattices'. Algebra Universalis 3 (1973), 294-303.

[9] G. Czédli, A. P. Huhn and E. T. Schmidt, 'Weakly independent subsets in lattices'. Algebra Universalis 20 (1985), 194-196.

[10] B. A. Davey and H. A. Priestley, Introduction to Lattices and Order (Cambridge University Press. Cambridge. 1990).

[11] J. Hashimoto, 'Ideal theory for lattices', Math. Japon. 2 (1952). 149-186.

[12] K. Kuratowski, Topologie, I (Monogr. Mat., T. III, Warsaw, 1933).

[13] Z. Lengvárszky, Independent Subsets in Lattices (Ph.D. Thesis, University of South Carolina. 1996).

[14] R. McKenzie, 'Equational bases and non-modular lattice varieties', Trans. Amer. Math. Soc. 174 (1972), 1-43.

[15] E. H. Moore, Introduction to a form of general analysis, Amer. Math. Soc. Colioq. Publ. 2 (Amer. Math. Soc.. Providence, RI, 1910).

[16] O. Ore, 'Combinations of closure operators', Ann. Math. 44 (1943), 514-533.

[17] _ 'Some studies on closure relations'. Duke Math. J. 10 (1943), 761-785.

[18] I. Rival. 'Maximal sublattices of finite distributive lattices'. Proc. Amer. Math. Soc. 37 (1973). $417-420$. 
[19] _ _ 'Maximal sublattices of finite distributive lattices, II', Proc. Amer. Math. Soc. 44 (1974), 263-268.

[20] C. Ryter and J. Schmid, 'Deciding Frattini is NP-complete', Order 11 (1994), 257-279.

[21] J. Tủma, 'On simultaneous representations of distributive lattices', Acta Sci. Math. (Szeged) 58 (1993), 67-74.

[22] F. Vogt, 'Bialgebraic contexts for finite distributive lattices', Algebra Universalis 35 (1996), 151165.

[23] M. Ward, 'The closure operators of a lattice', Ann. Math. 43 (1942), 191-196.

Zsolt Lengvárszky

Department of Computer Science

University of South Carolina

Columbia, SC 29208. USA

e-mail: zlengvar@cs.sc.edu
George F. McNulty Department of Mathematics University of South Carolina Columbia, SC 29208. USA

e-mail: mcnulty@math.sc.edu 